\title{
Special Issue: In Honor of Sara S. Sparrow, Ph.D.
}

\author{
Fred R. Volkmar • Elena Grigorenko
}

Published online: 5 January 2014

(c) Springer Science+Business Media New York 2014

The articles in this special section were written to honor the memory of Sara S. Sparrow, Ph.D., a leader in the field of developmental disabilities and psychological assessment. As a teacher, mentor, colleague, and friend Sara profoundly influences the careers of many junior colleagues. The author of more than 100 articles and chapters in the fields of psychological assessment and developmental disabilities Sara was also the author of the Vineland Adaptive Behavior Scales (1984), one of the most widely used psychological assessment instruments in the world.

The articles in this section reflect the diversity of her interests in developmental disabilities. Fletcher and Morris focus on issues of reading, laterality and the brain and neuropsychological models of reading disabilities. Green and Carter focus on the relations of daily living skills in young children with autism in relation to parental stress. Their sophisticated analyses illustrate the importance of including an explicit focus on adaptive skills in intervention program. Rhea Paul and colleagues also highlight the importance of daily living skills in very young children with autism relative to both severity of autistic symptoms and overall nonverbal ability. Tan, Reich, Hart, Thuma, and Grigorenko's articles focuses on another aspect of adaptive skills in relation to achievement in a rural African community and their potential relationships to outcome. Ventola, Saulnier, Steinberg, Chawarska, and Klin report the results of an item analysis of the Vineland and those behaviors which most robustly differentiate children with ASD from those with intellectual disability. Dykens focuses on leisure time activities in individuals with PraderWilli Syndrome and their relationship to cognitive and adaptive abilities. Finally Domenic Cicchetti, Sara's spouse and long time collaborator, closes this section with a discussion of scales of measure in ASD and their relation to validity and reliability.

An active clinician, teacher and researcher Dr. Sparrow's work touched the lives of thousands of children, her many students and trainees, and a number of colleagues at Yale and other institutions. The articles in this section reflect the diversity of her interests and her commitment to bettering the lives of all those with developmental vulnerabilities. 